CARDIOVASCULAR MEDICINE

\title{
Sex differences in the expression of haemorheological determinants in individuals with atherothrombotic risk factors and in apparently healthy people
}

\author{
D Zeltser, O Rogowski, S Berliner, T Mardi, D Justo, J Serov, M Rozenblat, D Avitzour, I Shapira
}

Heart 2004;90:277-281. doi: 10.1136/hrt.2003.014753

See end of article for authors' affiliations

Correspondence to:

Professor Shlomo Berliner, Department of Medicine "D", Tel-Aviv Sourasky Medical Centre, 6 Weizman Street, Tel Aviv 64239, Israel; shapiraiz@ tasmc.health.gov.il

Accepted 29 June 2003
Background: Increased red cell aggregation can be detrimental, leading to slow capillary blood flow and tissue hypoxaemia. Sex differences in the degree of erythrocyte adhesiveness/aggregation in the peripheral blood have not been clearly shown.

Objectives: To determine whether there are sex differences in the expression of erythrocyte adhesiveness/ aggregation in the peripheral blood in individuals with atherothrombotic risk factors and in apparently healthy people.

Methods: From a cohort of 965 participants in the Tel Aviv Medical Centre inflammation survey, 192 pairs of different sex were matched for age, body mass index, hip and waist circumferences, cardiovascular risk factors, and the intake of active cardiovascular drugs.

Results: Women had an enhanced degree of red cell aggregation $(p<0.0005)$ as well as increased concentrations of inflammation sensitive proteins including fibrinogen and $\mathrm{C}$ reactive protein. Women had a lower haemoglobin concentration than men, but this did not affect the degree of erythrocyte adhesiveness/aggregation.

Conclusions: The significant increase in red blood cell adhesiveness/aggregation in the peripheral blood of women with atherothrombosis could be relevant to the more eventful course that some women experience during and following acute ischaemic disease. l: has been shown repeatedly that enhanced red blood cell aggregation is associated with slow capillary blood flow, disturbed microcirculatory blood flow, and tissue hypoxaemia. ${ }^{1-6}$ Therapeutic interventions directed at reducing this deleterious haemorheological effect might attenuate the tendency for cells to adhere to each other and aggregate, thus improving a patient's clinical condition. ${ }^{78}$

Studies in the past did not take into account the potential differences between women and men regarding red blood cell adhesiveness/aggregation. Such differences could have an effect on the different prognosis of acute vascular events between women and men. ${ }^{9-11}$ In this study we show that there are significant differences in the degree of erythrocyte adhesiveness/aggregation in the peripheral venous blood between women and men. We discuss the relevance of this for potential microcirculatory obstruction under conditions of vascular occlusion and for the phenomenon of non-reperfusion.

\section{METHODS}

\section{Study population}

This cross sectional study involved participants from the following sources:

- apparently healthy employees of the Tel Aviv Medical Centre and Tel Aviv Municipality (Israel), including members of the medical staff and retired employees

- individuals with atherothrombotic risk factors who were being followed up in various outpatient clinics of the medical centre, including clinics for diabetes, hypertension, and metabolic disorders

- people with a history of a clinically overt vascular disease, including a history of coronary artery bypass grafting, myocardial infarction, cerebrovascular accident, or peripheral artery occlusive disease
- individuals who had been evaluated in our outpatient health screening programme.

All the subjects included in the survey gave their written informed consent according to the instructions of the local ethics committee. Recruitment was based on local announcements and advertisements in the monthly payroll bill of the medical personnel, as well as an appeal to the patients in the various outpatient clinics to participate in the inflammation survey.

We excluded any individuals with an underlying inflammatory disease (arthritis, inflammatory bowel disease, and so on) as well as those with any infection or other inflammatory condition, including infarction, surgery, or angiography, during the six months preceding the recruitment into the present study. We also excluded any individual treated with steroids or non-steroidal anti-inflammatory drugs, except for aspirin at doses less than $325 \mathrm{mg} /$ day. This dose was chosen because we have found that the intake of up to $325 \mathrm{mg}$ of aspirin a day does not have a significant effect on the degree of erythrocyte adhesiveness/aggregation (unpublished data).

\section{Population study design}

From the whole cohort of subjects, we tried as far as possible to match pairs of different sex with regard to age, body mass index (BMI), hip and waist circumferences, and cardiovascular risk factors, in decreasing order of importance. In order to do this, we first excluded any individual with a history of clinically overt vascular disease, as mentioned above, or any woman treated with hormonal replacement therapy, owing to its proven effect on $\mathrm{C}$ reactive protein concentrations and possibly on inflammation. We then excluded any individual with missing values of any of the anthropometric variables or inflammatory indices (high sensitivity $\mathrm{C}$ reactive protein (hsCRP) and fibrinogen). 


\section{Definition of risk factors score}

Diabetes mellitus was defined as a blood glucose concentration of $\geqslant 7 \mathrm{mmol} / \mathrm{l}$ fasting or $>11 \mathrm{mmol} / \mathrm{l}$ random on two separate occasions, or being treated with insulin or oral antidiabetic agents. Arterial hypertension was defined as a blood pressure of $\geqslant 140 / 90 \mathrm{~mm} \mathrm{Hg}$ or being on antihypertensive drugs. Hyperlipidaemia was defined as having a low density lipoprotein (LDL) cholesterol concentration of $\geqslant 3.4 \mathrm{mmol} / \mathrm{l}$ or a triglyceride concentration of $>1.8 \mathrm{mmol} / \mathrm{l}$, or being on HMG-CoA reductase inhibitors or fibrates.

\section{Laboratory variables}

The white blood cell count and differential count were done using a Coulter STKS automatic cell analyser (Beckman Coulter, Nyon, Switzerland); the erythrocyte sedimentation rate (ESR) by Westergren's method, ${ }^{12}$ fibrinogen concentration by the method of Clauss ${ }^{13}$ using a Sysmex 600 autoanalyser (Sysmex Corporation, Hyaga, Japan); and hsCRP by Rifai's method ${ }^{14}$, using a Behring BN II nephelometer (DADA Boehringer, Marburg, Germany).

\section{The Erythrosense biomarker}

This involved a simple slide test. ${ }^{15}$ Venous blood from the antecubital vein was obtained between 08.00 and 11.00 hours following an overnight fasting. Blood was drawn into a syringe containing sodium citrate (one volume of $3.8 \%$ sodium citrate to three volumes of whole blood). One drop of the citrated whole blood was trickled (from a height of $3 \mathrm{~cm}$ ) onto a slide inclined at an angle of $30^{\circ}$ and allowed to run down by gravity, leaving a fine film. The slides were left to dry in that position, at room temperature. A technician who was blinded to the clinical and laboratory results of the patients scanned the slides using an image analysis system (Inflamet, Inflamet Co, Tel Aviv, Israel). ${ }^{16}$

\section{The inflammation meter (Inflamet)}

This system consists of a personal computer equipped with a Matrox Meteor colour frame grabber (Matrox Co, Montreal, Canada), a colour charge couple device (CCD) camera, and a microscope which was operated at $200 \times$ magnification, resulting in an image resolution of 0.4 micron per pixel. ${ }^{17}$ Nine images were taken from each slide. The fields of view were chosen systematically to sample different regions on the slide. Each image is processed separately and the outputs are then averaged to form the final slide outputs. The nine fields of view cover a total area of $0.6 \mathrm{~mm}^{2}$. A variable called the erythrocyte percentage (EP) was chosen to represent the degree of red blood cell adhesiveness/aggregation. This is essentially a measurement of the area that is covered by the red blood cells. In the absence of aggregation the covered area is $100 \%$, whereas with the appearance of enhanced erythrocyte adhesiveness/aggregation this percentage is lower. Thus the higher the degree of aggregation, the lower is the area covered by the cells, and vice versa. This is because during cell aggregation, free spaces are formed between the aggregated cells. These free spaces are subtracted from total slide area covered by red blood cells. A typical example is represented in fig 1 .

\section{Inflamet variabilities}

The coefficient of variation for the erythrocyte aggregation/ adhesiveness test (EAAT)—when one person prepared and read the different slides of a patient with inflammation-was 0.14 . We repeated this evaluation in five different patients with the same results. When nine different people prepared slides from the same patient the coefficient was 0.07. It was 0.1 when the same person read the same slide 10 times. The interobserver variability of this test was discussed by us in a study done on 273 individuals with various degrees of infection/inflammation. ${ }^{18}$ We found a substantial interobserver concordance which was at least as good as that obtained when a chest $x$ ray is shown to different specialists in radiology. In addition, we have recently reported the day to day variation in EAAT in a group of 30 individuals who had repeated EAAT determinations (between seven and 13 examinations per patient). In that study, we were able to show that the daily fluctuations in the test are comparable to other commonly used variables of the acute phase response, including $C$ reactive protein, white blood cell count, ESR, and fibrinogen concentrations. ${ }^{19}$ In addition, a highly significant correlation $(r=0.94, \mathrm{n}=50, \mathrm{p}<0.001)$ was obtained when the same slide was analysed by two independent individuals (unpublished data).

\section{Statistical analysis}

All continuous variables are summarised and displayed as mean (SD) for each sex separately, and the categorical data are summarised and displayed as the number of persons in each group plus the percentage in the group. For continuous variables, a comparison between the sexes was made using Student's $t$ test for paired samples. For all the categorical variables the paired sign test was used to test the possible difference between the groups. In order to reject the hypothesis that the lower haemoglobin concentration in women contributes to their enhanced erythrocyte adhesiveness/aggregation, we calculated haemoglobin adjusted Pearson's correlation coefficients between the results of the Erythrosense biomarker and the inflammatory variables hsCRP and fibrinogen, and compared them with the nonhaemoglobin adjusted correlation. In addition we calculated different linear regression models to assess the contribution of haemoglobin, hs-CRP, and fibrinogen to the prediction of the degree of the erythrocyte adhesiveness/aggregation. In both the correlations and the linear regression, a logarithmic

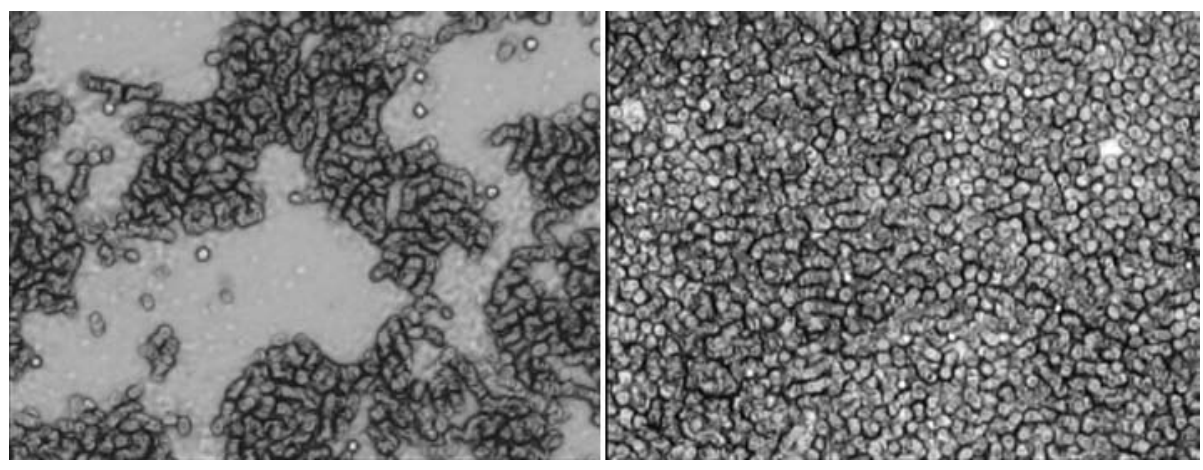

Figure 1 A typical example of a patient with ischaemic heart disease and enhanced degree of erythrocyte adhesiveness/aggregation (left) and a control (right). 
Table 1 Basic characteristic including the anthropometric variables

\begin{tabular}{llll}
\hline & Women $(\mathbf{n}=192)$ & Men $(\mathbf{n}=192)$ & p Value* \\
\hline Age (years) & $48.8(11.7)$ & $48.7(11.9)$ & NS \\
BMI $\left(\mathrm{kg} / \mathrm{m}^{2}\right)$ & $30.1(5.7)$ & $30.1(5.6)$ & NS \\
Waist $(\mathrm{cm})$ & $99.6(13.5)$ & $105.4(13.0)$ & $<0.0005$ \\
Hip $(\mathrm{cm})$ & $105.9(10.7)$ & $105.0(10.5)$ & NS \\
Waist/hip ratio & $0.94(0.11)$ & $1.00(0.08)$ & $<0.0005$ \\
\hline
\end{tabular}

Values are mean (SD); *significance was determined by paired $t$ test between the sexes.

BMI, body mass index.

transformation was used in hs-CRP and fibrinogen because of their non-normal distribution.

All analyses were carried out using SPSS software (SPSS Inc, Chicago, Illinois, USA).

\section{RESULTS}

There were 852 individuals (420 men and 432 women) who met all the inclusion criteria. We were able to match 192 pairs on the basis of age and anthropometric variables. It can be seen that although full match was achieved for age, BMI, and hip circumference, waist circumference was significantly greater among the men; as a result the waist/hip ratio was also higher in men (table 1 ).

Pairs of women and men were matched for their atherothrombotic risk factors (table 2). We calculated the mean number of risk factors per individual and found a perfect match (table 2 ). In addition, we recorded the number and percentage of individuals who were on cardiovascular drugs that might have effects on the inflammatory response or on variables of potential rheological significance (table 3 ). There was a perfect match between the pairs (table 3 ) except for intake of aspirin and $\alpha$ blockers, which was greater in men.

Finally we report the results of the degree of erythrocyte adhesiveness/aggregation (expressed as the erythrocyte percentage, EP) as well as those relating to lipid and inflammatory variables (table 4). An enhanced degree of erythrocyte adhesiveness/aggregation was found in the women compared with the men, along with increased concentrations of the inflammation sensitive proteins hsCRP and fibrinogen. In addition, the women had a lower haemoglobin concentration than the men, along with higher concentrations of high density lipoprotein and lower concentrations of triglycerides (table 4).

In order to reject the possibility that enhanced erythrocyte adhesiveness/aggregation reflects the presence of anaemia in women, we did a linear regression analysis between haemoglobin, fibrinogen, and hs-CRP in their contribution to the degree of erythrocyte adhesiveness/aggregation. We found that in all possible models in both sexes, haemoglobin was excluded from effects on erythrocyte adhesiveness/

Table 2 Number and percentage of atherothrombotic risk factors in women and men matched for age and anthropometric variables

\begin{tabular}{llll}
\hline Atherothrombotic risk factor & Women & Men & p Value* \\
\hline Smoker & $66(34.4 \%)$ & $78(40.6 \%)$ & $\mathrm{NS}$ \\
Diabetes mellitus & $11(5.7 \%)$ & $7(3.6 \%)$ & $\mathrm{NS}$ \\
Hyperlipidaemia & $59(30.7 \%)$ & $47(24.5 \%)$ & $\mathrm{NS}$ \\
$\begin{array}{l}\text { Hypertension } \\
\text { Mean (SD) number of risk factors }\end{array}$ & $28(14.6 \%)$ & $39(20.3 \%)$ & $\mathrm{NS}$ \\
per individual & $0.85(0.84)$ & $0.89(0.83)$ & $\mathrm{NS}$ \\
\hline *Paired $t$ test. & & & \\
\hline
\end{tabular}

Table 3 Number and percentage of individuals taking cardiovascular drugs that might have an effect on the inflammatory response or have haemorheological relevance

\begin{tabular}{llll}
\hline Drug & Women & Men & p Value* $^{*}$ \\
\hline Aspirin & $7(3.6 \%)$ & $19(9.9 \%)$ & 0.023 \\
Nitrates & $0(0 \%)$ & $1(0.5 \%)$ & NS \\
Amiodarone & $0(0 \%)$ & $1(0.5 \%)$ & NS \\
$\alpha$ Blockers & $0(0 \%)$ & $7(3.6 \%)$ & 0.016 \\
$\beta$ Blockers & $13(6.8 \%)$ & $12(6.3 \%)$ & NS \\
Calcium channel blockers & $7(3.6 \%)$ & $15(7.8 \%)$ & NS \\
ACE inhibitors & $8(4.2 \%)$ & $13(6.8 \%)$ & NS \\
Angiotensin II receptor blocker & $4(2.1 \%)$ & $2(1.0 \%)$ & NS \\
HMG-CoA reductase blockers & $9(4.7 \%)$ & $5(2.6 \%)$ & NS \\
Fibrates & $3(1.6 \%)$ & $2(1.0 \%)$ & NS
\end{tabular}

*Paired $t$ test.

$\mathrm{ACE}$, angiotensin converting enzyme; HMG-CoA, hydroxymethyl glutaryl coenzyme A.

aggregation, as it made a non-significant contribution to the model (data not shown). Pearson's correlation adjusted for haemoglobin concentration showed no significant difference between the sexes. Thus haemoglobin concentration did not contribute to the enhanced degree of erythrocyte adhesiveness/aggregation in women (table 5).

\section{DISCUSSION}

It has been shown in the past that there are sex specific differences in cardiovascular mortality, to the detriment of women. ${ }^{9-11}{ }^{20}$ The possibility that enhanced inflammation and red blood cell adhesiveness/aggregation in women might contribute to a worse haemorheological profile has not been considered in the past. This could be relevant to the pathophysiology of microcirculatory flow in conditions of vascular obstruction, occlusion of small vessels, slow flow, and cell stagnation within the capillary network. ${ }^{1-6}$ Indeed it has been shown that enhanced red cell aggregation can contribute to tissue hypoxaemia. ${ }^{4}$

We have recently adopted a whole blood slide test and image analysis to quantify the tendency of red blood cells to adhere to each other and aggregate in the peripheral venous blood. This Erythrosense biomarker is based on the known

Table 4 Comparison between women and men of erythrocyte adhesiveness/aggregation (expressed as erythrocyte percentage) together with lipid and inflammatory variables

\begin{tabular}{|c|c|c|c|}
\hline Variable & Women & Men & p Value* \\
\hline EP (\%) & $79.1(15.0)$ & $91.4(10.3)$ & $<0.0005$ \\
\hline $\operatorname{ESR}(\mathrm{mm} / \mathrm{h})$ & $26.3(15.5)$ & $13.3(10.8)$ & $<0.0005$ \\
\hline hs-CRP (mg/l) & $5.8(7.4)$ & $3.8(6.3)$ & 0.004 \\
\hline Fibrinogen (mg/dl) & $323(60)$ & $288(60)$ & $<0.0005$ \\
\hline WBCC $\left({ }^{*} 10^{3}\right.$ cells $\left./ \mu \mathrm{l}\right)$ & $7.36(2.00)$ & $7.06(1.89)$ & NS \\
\hline PMNC $\left({ }^{*} 10^{3}\right.$ cells $\left./ \mu \mathrm{l}\right)$ & $4.39(1.58)$ & $4.25(1.51)$ & NS \\
\hline Monocytes $\left({ }^{*} 10^{3}\right.$ cells $\left./ \mu \mathrm{l}\right)$ & $0.54(0.38)$ & $0.56(0.16)$ & NS \\
\hline Haemoglobin (g/dl) & $13.0(0.99)$ & $14.9(0.9)$ & $<0.0005$ \\
\hline $\operatorname{MCV}\left(\left.\mu\right|^{3}\right)$ & $86.0(5.2)$ & $86.9(4.1)$ & NS \\
\hline Total cholesterol (mmol/l) & $5.74(1.06)$ & $5.69(1.11)$ & NS \\
\hline LDL cholesterol (mmol/l) & $3.54(0.9)$ & $3.62(0.96)$ & NS \\
\hline HDL cholesterol (mmol/li) & $1.45(0.39)$ & $1.34(0.31)$ & $<0.0005$ \\
\hline Triglycerides (mmol/l) & $1.64(0.97)$ & $1.87(1.81)$ & 0.033 \\
\hline
\end{tabular}

Values are mean (SD).

*Paired $t$ test.

$E P$, erythrocyte percentage; $E S R$, erythrocyte sedimentation rate; $H D L$, high density lipoprotein; hs-CRP, high sensitivity $C$ reactive protein; LDL, low density lipoprotein; MCV, mean corpuscular volume; PMN, polymorphonuclear leucocyte count; WBCC, white blood cell count. 
Table 5 Pearson's correlation coefficients, with and without an adjustment for haemoglobin, between fibrinogen or hs-CRP and the degree of erythrocyte adhesiveness/ aggregation, expressed as the erythrocyte percentage

\begin{tabular}{|c|c|c|c|c|c|}
\hline & & \multicolumn{2}{|l|}{ Women } & \multicolumn{2}{|l|}{ Men } \\
\hline & & Not adjusted & $\mathrm{Hb}$ adjusted & Not adjusted & $\mathrm{Hb}$ adjusted \\
\hline $\begin{array}{l}\text { EP } v \log h s-C R P \\
\text { EP } v \log \text { fibrinogen }\end{array}$ & $\begin{array}{l}\text { Correlation } \\
\text { Significance } \\
\text { Correlation } \\
\text { Significance }\end{array}$ & $\begin{array}{l}-0.43 \\
<0.0005 \\
-0.51 \\
<0.0005\end{array}$ & $\begin{array}{l}-0.42 \\
<0.0005 \\
-0.53 \\
<0.0005\end{array}$ & $\begin{array}{l}-0.49 \\
<0.0005 \\
-0.52 \\
<0.0005\end{array}$ & $\begin{array}{l}-0.47 \\
<0.0005 \\
-0.50 \\
<0.0005\end{array}$ \\
\hline
\end{tabular}

$\mathrm{Hb}$, haemoglobin; $E P$, erythrocyte percentage; $h s-C R P$, high sensitivity $C$ reactive protein.

observations that multiple inflammation sensitive proteinsincluding fibrinogen, immunoglobulins, haptoglobin, caeruloplasmin, $\alpha-1$ acidic protein, and hs-CRP-are associated with the induction or maintenance of enhanced red blood cell aggregation..$^{21-24}$ Thus our biomarker uses the erythrocyte as a sensor for the presence of these adhesive macromolecules in the plasma milieu around and between the cells. The resulting degree of cell adhesiveness/aggregation is probably a summarised effect of these proteins on the tendency of the cells to adhere to each other and aggregate. We have already shown that enhanced red blood cell adhesiveness/aggregation correlates significantly with the presence of enhanced fibrinogen concentrations ${ }^{25}$ and with the degree of the patient's inflammatory response, ${ }^{26}$ that it can be induced by the intravenous infusion of immunoglobulins, ${ }^{27}$ and that it is abolished by plasmapheresis. ${ }^{28}$

The possibility that there might be sex differences in the tendency of red blood cells to adhere to each other and aggregate has not been evaluated in a systematic way in the past. Our study is the first to match women and men for multiple factors that might affect the inflammatory response, the concentrations of inflammation sensitive proteins, and the degree of red cell adhesiveness/aggregation. These include age, obesity, atherothrombotic risk factors, and vascular events, as well as the intake of therapeutic drugs. The results are significant in that they show a clear sex difference in the degree of red cell adhesiveness/aggregation. The difference is probably related to the enhanced inflammatory response and the presence of increased fibrinogen concentrations, and not to a lower haemoglobin concentration in women.

An adverse outcome in individuals with enhanced inflammation and erythrocyte adhesiveness/aggregation has been shown by us in several small cohorts in the past. ${ }^{29-31}$ It is known that increased concentrations of fibrinogen may have harmful rheological implications. ${ }^{72}$ In addition, a heightened baseline inflammatory profile, including increased $\mathrm{C}$ reactive protein, could contribute to an adverse vascular course in individuals with acute ischaemic diseases..$^{33}$ Thus the enhanced erythrocyte adhesiveness/aggregation that is found in women with inflammation and increased concentrations of inflammation sensitive proteins could be a factor in the pathophysiology of cell stagnation in occluded microvessels. A better understanding of these pathophysiological events should lead to therapeutic interventions to reduce inflammation and improve the rheological profile.

A potential effect of hormonal therapy has been noted by other investigators in the past. ${ }^{34} 35$ None of the 81 postmenopausal women in this study was on hormonal replacement therapy, and only 10 of the 111 premenopausal women were on oral contraceptives. Analysis of the premenopausal women who were not on contraceptives did not alter the results reported here. However, our study was not designed to address the issue of the potential effect of hormonal replacement therapy or oral contraceptives on the haemorheological variables.

\section{Conclusions}

There are sex differences with respect to the degree of inflammation, the concentrations of inflammation sensitive protein, and red blood cell adhesiveness/aggregation in the peripheral blood. A better understanding of these sex differences could pave the way for a more potent antiinflammatory approach to treating women at risk of future vascular events.

\section{Authors' affiliations}

D Zeltser, O Rogowski, S Berliner*, T Mardi, D Justo, J Serov, M Rozenblat, I Shapira, Department of Internal Medicine " $D$ ", Tel Aviv Sourasky Medical Centre, Tel Aviv University, Tel Aviv, Israel

D Avitzour, Timorim Technologies, Jerusalem, Israel

*Professor Berliner is a shareholder of Inflamet Ltd, Tel Aviv, Israel

\section{REFERENCES}

1 Soutani M, Suzuki Y, Tateishi N, et al. Quantitative evaluation of flow dynamics of erythrocytes in microvessels: influence of erythrocyte aggregation. Am J Physiol 1995;268:H1959-65.

2 Schmid-Schonbein H. Blood rheology and physiology of microcirculation. Ric Clin Lab 1981;11(suppl 1):13-33.

3 Bishop JJ, Nance PR, Popel AS, et al. Effect of erythrocyte aggregation on velocity profiles in venules. Am J Physiol Heart Circ Physiol 2001;280:H222-36.

4 Tateishi N, Suzuki Y, Cicha I, et al. $\mathrm{O}_{2}$ release from erythrocytes flowing in a narrow $\mathrm{O}_{2}$-permeable tube: effects of erythrocyte aggregation. Am J Physiol Heart Circ Physiol 2001;281:H448-56.

5 Mchedlishvili G, Gobejishvili L, Mamaladze A, et al. Microcirculatory stasis induced by hemorheological disorders: further evidence. Microcirculation 1999;6:97-106

6 Cabel M, Meiselman HJ, Popel AS, et al. Contribution of red blood cell aggregation to venous vascular resistance in skeletal muscle. Am J Physiol 1997;272:H1020-32

7 Suckfull M. Fibrinogen and LDL apheresis in treatment of sudden hearing loss: a randomised multicentre trial. Lancet 2002;360:1811-17.

8 Klingel R, Fassbender C, Fassbender T, et al. Rheopheresis: rheologic, functional and structural aspects. Ther Apher 2000;4:348-57.

9 Khan SS, Nessim S, Gray R, et al. Increased mortality of women in coronary artery bypass surgery: evidence for referral bias. Ann Intern Med 1990;1 12:561-7.

10 Tofler GH, Stone PH, Muller JE, et al. Effects of gender and race on prognosis after myocardial infarction: adverse prognosis for women, particularly black women. J Am Coll Cardiol 1987;9:473-82.

11 Greenland P, Reicher-Reiss H, Goldbourt U, et al. In-hospital and 1-year mortality in 1524 women after myocardial infarction. Comparison with 4315 men. Circulation 1991;83:484-91.

12 International Committee for Standardization in Hematology. Recommendation of measurement of erythrocyte sedimentation rate of human blood. Immunochemistry 1965;2:235-54.

13 Clauss A. Gerinnungsphysiologische Schnellmethode zur Bestimmung des Fibrinogens. Acta Haematol Basel 1957;17:237-46.

14 Rifai N, Tracy RP, Ridker PM. Clinical efficacy of an automated high-sensitivity C-reactive protein assay. Clin Chem 1999;45:2136-41.

15 Rotstein R, Landau T, Twig A, et al. The erythrocyte adhesiveness/ aggregation test (EAAT). A new biomarker to reveal the low grade subclinical smoldering inflammation individuals with atherosclerotic risk factors. Atherosclerosis 2002;165:343-51.

16 Fusman G, Mardi T, Justo D, et al. Red blood cell adhesiveness/aggregation, C-reactive protein, fibrinogen and erythrocyte sedimentation rate in healthy adults and in those with atherosclerotic risk factors. Am J Cardiol 2002;90:561-3. 
17 Gamzu R, Rotstein R, Fusman R, et al. Increased erythrocyte adhesiveness and aggregation in peripheral venous blood of women with pregnancy-induced hypertension. Obstet Gynecol 2001;98:307-12.

18 Rotstein R, Fusman R, Zeltser D, et al. The picture of inflammation: a new concept that combines the white blood cell count and erythrocyte sedimentation rate into a new hematologic diagnostic modality. Acta Haematol 2001;106:106-14.

19 Sharshun Y, Brill S, Mardi T, et al. Inflammation at a glance. The erythrocyte adhesiveness/aggregation to reveal the presence of inflammation in individuals with atherothrombosis. Heart Dis 2003;5:182-3.

20 Vaccarino V, Parsons L, Every NR, et al. Sex-based differences in early mortality after myocardial infarction. National registry of myocardial infarction 2 participants. N Engl J Med 1999;341:217-25.

21 Weng X, Cloutier G, Beaulieu R, et al. Influence of acute-phase proteins on erythrocyte aggregation. Am J Physiol 1996;271:H2346-52.

22 Weng X, Roederer GO, Beaulieu R, et al. Contribution of acute-phase proteins and cardiovascular risk factors to erythrocyte aggregation in normolipidemic and hyperlipidemic individuals. Thromb Haemost 1998;80:903-8.

23 Talstad I, Scheie P, Dalen H, et al. Influence of plasma proteins on erythrocyte morphology and sedimentation. Scand J Haematol 1983;31:478-84.

24 Imaizumi K, Shiga T. Effect of immunoglobulins and lgG-fragments on the human erythrocyte aggregation, studied by rheoscope combined with image analyzer. Biorheology 1983;20:569-77.

25 Rogowski O, Zeltser D, Rotstein R, et al. Correlated expression of adhesive properties for both white and red blood cells during inflammation. Biorheology 2000;37:361-70.

26 Rotstein R, Zeltser D, Shapira I, et al. The usefulness of an inflammation meter to detect the presence of infection/inflammation in elderly patients. J Gerontol A Biol Sci Med Sci 2002;57:M122-7.
27 Fusman R, Zeltser D, Rotstein R, et al. INFLAMET: an image analyzer to display erythrocyte adhesiveness/aggregation. Eur J Intern Med 2000;11:271-6.

28 Hershcovici T, Elishkewitch $\mathrm{K}$, Rotstein $\mathrm{R}$, et al. The erythrocyte adhesiveness/ aggregation test to reveal real-time information of rheological relevance in patients with familial and primary hypercholesterolemia before and following plasma exchange. Ther Apher 2002;6:140-4.

29 Berliner S, Rotstein R, Fusman R, et al. Increased erythrocyte adhesiveness/aggregation in the peripheral venous blood of patients with ischemic heart disease and an eventful course. Acta Cardiol 2001;56:121-6.

30 Zeltser D, Bornstein NM, Rotstein R, et al. The erythrocyte adhesiveness/ aggregation test in the peripheral blood of patients with ischemic brain events. Acta Neurol Scand 2001;103:316-19.

31 Anuk T, Ben Assayag E, Rotstein R, et al. Prognostic implications of admission inflammatory profile in acute ischemic neurological events. Acta Neurol Scand 2002;106:166-99.

32 Maresca G, Di-Blasio A, Marchioli R, et al. Measuring plasma fibrinogen to predict stroke and myocardial infarction: an update. Arterioscler Thromb Vasc Biol 1999:19:1368-77.

33 Folsom AR, Aleksic N, Catellier D, et al. C-Reactive protein and incident coronary heart disease in the atherosclerosis risk in communities (ARIC) study. Am Heart J 2002;144:233-8.

34 Demiroglu H, Barista I, Dundar S. The effect of age and menopause on erythrocyte aggregation. Thromb Haemost 1997;77:403-7.

35 Goncalves I, Saldanha C, Martins e Silva J. Beta-estradiol effect on erythrocyłe aggregation-a controlled in vitro study. Clin Hemorheo Microcirc 2001;25:127-34.

\section{IMAGES IN CARDIOLOGY}

\section{Non-compaction of the left ventricle}

$\mathrm{N}$ on-compaction of the left ventricle is a rare congenital cardiomyopathy characterised by a loosened spongy myocardium. The disorder may be associated with facial dysmorphism and familial recurrence and is caused by intrauterine arrest of compaction of the loose interwoven meshwork.

Left ventricular angiography (below left), cardiac magnetic resonance imaging (MRI) (below centre), and echocardiography (below right) typically reveal a dilated hypocontractile left ventricle with a two tailored wall; the inner zones of heavily spongious, trabecularised endocardial layers with deep intertrabecular recesses can be distinguished from thin outer zones of compacted myocardium. Histological examination confirms the spongy appearance with deep intertrabecular recesses, lined by endothelium, which spread close to the epicardial surface.

Prompt recognition of the disease is mandatory because of its high mortality and morbidity. Heart failure, thromboembolic events, and ventricular arrhythmias all have been reported. Besides familial screening and risk stratification, treatment is directed to prevent and manage heart failure, ventricular arrhythmias, and cardiac thromboembolism.

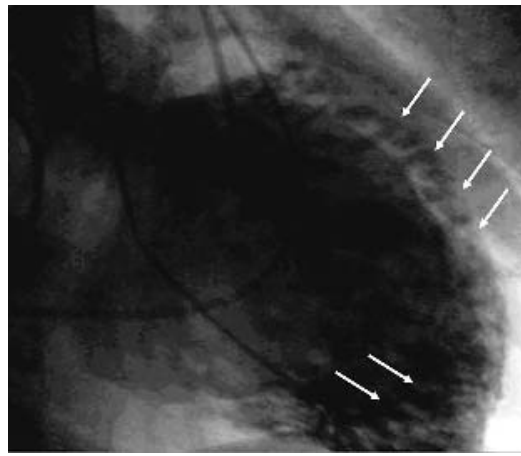

Left ventricular angiography, $30^{\circ}$ right anterior oblique projection. Arrow indicates noncompacted myocardium.

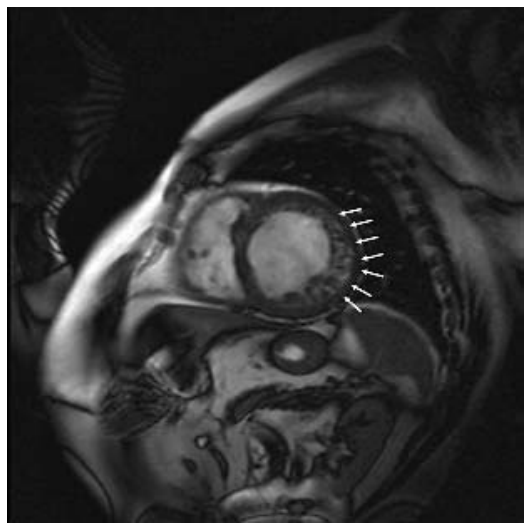

MRI double inversion recovery images in diastole in left ventricular short axis view. Arrows indicate thickened non-compacted myocardial wall.

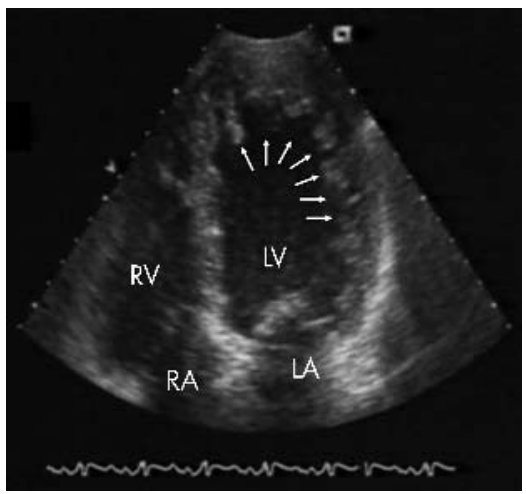

Echo apical long axis view. Arrows indicate non-compacted left ventricle. LV, left ventricle; $\mathrm{RV}$, right ventricle; LA, left atrium; RA, right atrium. 